\title{
Commentary on the IV São Paulo Research Conference: Cancer Today: from molecular biology to treatment, São Paulo, SP, Brazil, November 10-12, 2005
}

E. Mihich

Correspondence

E. Mihich

Roswell Park Cancer Institute

Buffalo, NY

USA

Received June 23, 2006
Roswell Park Cancer Institute, Buffalo, NY, USA
This symposium focused on basic aspects of molecular mechanisms of cancer cell survival and also on epidemiological and immunological factors, which would provide for the development of new treatments and other modalities of intervention. It was an outstanding symposium attesting to the excellence of oncological sciences in Brazil and to their invaluable contribution to the international efforts directed towards the control of cancer. Through the participation of several international leaders in oncology the symposium also provided evidence for how well integrated Brazilian science is with the most avant-garde scientific activities in Europe and in the US. Finally, the active participation of a large number of young scientists and graduate students held an irrefutable promise for the brilliant future of the Community of Brazilian scientists devoted to studies of the molecular biology and treatment of cancer.

Several institutions from São Paulo, Rio de Janeiro and other parts of the country were represented through the presentation of lectures and posters. Undoubtedly, the Ludwig Institute for Cancer Research, the associated Hospital do Câncer A.C. Camargo in São Paulo, and the Instituto Nacional do Câncer (INCa) in Rio de Janeiro were "first among equals" together with other centers in the quality of their contributions. The diversified expertise of participants coming from excellent institutions in different parts of Brazil provided the opportunity for lively discussions stemming from consideration of the role of epigenetic and other basic molecular mechanisms of regulation of the cancer cell.

The meeting opened with a keynote address by Dr. R. Brentani, who outlined progress in cancer research in Brazil and at the Ludwig Institute. Key advances mentioned included, for 
instance, the clarification of the mechanisms of the antiapoptotic action of prions or of prion-mimicking peptides, particularly through their binding to stress-inducible protein and the consequent cooperation with HSP70 and HSP90; the role of epigenetic factors in conditioning genome instability with emphasis on differential methylation in chromosome 5; the discovery, in cooperation with scientists of the University of São Paulo, of the new gene FLJ in prostate tumors and its down-regulation by androgen isoforms, and the finding by Dr. L. Reis of PPM1D whose expression is induced by androgens. This presentation already provided a clear indication of the high quality of Brazilian science and its contributions at the cutting edge of oncology research.

Dr. E. Franco, now at McGill University and collaborating with Dr. L. Villa from the Ludwig Institute (São Paulo) and Dr. G. Azevedo from the Instituto Nacional do Câncer (Rio de Janeiro), discussed the opportunities provided by epidemiological approaches for assessing the role of cancer etiological factors. Dr. E. Franco emphasized the difference between focusing on one factor, e.g., smoking, versus other factors, e.g., diet, and also comparisons between developed and developing countries. He also indicated why the identification of etiological processes was a bit easier in the case of tumors associated with viruses like HBV or HPV. Dr. G. Azevedo stated that in Brazil lung cancer had not increased very much in females as a consequence of increases in smoking and emphasized the need to distinguish between primary and secondary studies of interactions among etiological factors; for example, multiple environmental factors versus genetic and epigenetic mechanisms of control of metabolic pathways. These two good presentations emphasized the value of epidemiological approaches in etiological studies but cautioned about the need to consider also the multiplicity of factors possibly involved.
The next session was focused on discussions of mechanisms of control of the cell cycle. Dr. H. Armelin, of the Instituto de Química, Universidade de São Paulo, discussed the apoptotic but also the oncogenic role of FGF2 and the fact that the apoptotic action appeared to be specific for tumor cells. FGF2 is one of the 23 members of the Fibroblast Growth Factor Family and the study was carried out in the Y1 adrenocortical tumor cell line which is P53-positive. Upon exposure to FGF2, these cells lose viability beyond S-phase initiation and do not reach mitosis. Resistance to FGF2 can develop in non-immunogenic cells. The dependence of apoptotic effects on ki-ras GTP was illustrated, as was the fact that resistance can occur also in the presence of high basal levels of ki-ras GTP but that cells with loss of amplified c-ki-ras sequences are resistant and non-tumorigenic. These were elegant studies by an expert group of scientists. Dr. J.-P. Viola, from the Instituto Nacional de Câncer, Rio de Janeiro, discussed the cell cycle regulation by transcription factor NFAT (nuclear factor of activated T cells). After describing $\mathrm{T}$ cell activation upon exposure to antigen he outlined the calcineurin-dependent activation of NFAT and the mechanisms of control of NFAT on lymphocyte proliferation and modification of cytokine levels. The negative regulatory regions on NFAT were studied and effects on cyclin A2, mutated cyclin A2 and IL-2 determined. Moreover, it was found that cyclosporin A inhibits in vivo the binding of NFAT2 to the promoter of cyclin A2 and also blocks the translocation of NFAT to the nucleus. In vivo the inhibition of lymphocyte proliferation by NFAT was prevented by cyclin A. It was further shown that NFAT2 is oncogenic whereas NFAT- 1 is a tumor suppressor in several experimental tumor models. These studies attested to the outstanding level of molecular immunology expertise in Brazil and contributed significantly to the understanding of the controls of 
immunological effectors.

The third session was focused on a discussion of tyrosine kinases as functional components of mechanisms of regulation of cancer cells and as specific targets for therapeutic intervention. In this session Dr. N. Hynes, from the Institute for Biomedical Research in Basel, Switzerland, Dr. O. Hermine from Hôpital Necker in France, Dr. C. Heldin, from the Ludwig Institute for Cancer Research in Uppsala, Sweden, and Dr. G. Groner, from the Georg Speyer Haus in Germany, presented important lectures and their participation in the meeting was tangible evidence of the collegial interactions between Brazil and international sciences. Dr. N. Hynes discussed the biology of Erb B-2 and possible intervention through the use of antibody or small molecule tyrosine kinase inhibitors and a basis for resistance to such inhibitors. Examples of synergism between EGF-R and VEGF inhibitors were illustrated as potential leads towards anticancer interventions. Dr. O. Hermine discussed the presence of c-kit mutations in mastocytosis and related cutaneous lesions. A c-kit transgenic mouse model was described in which mastocytosis develops and can be inhibited by Gleevec; indeed, this agent inhibits phosphorylated c-kit. The effects of other agents were also discussed. Dr. C. Heldin outlined the role of platelet-derived growth factor (PDGF) in embryo development, regulation of interstitial fluid pressure and wound healing. The involvement of PDGF in fibrotic conditions, atherosclerosis, cancers such as glioblastoma and sarcoma, and the dysregulation of PDGF-R in tumors were discussed. The use of aptemers specific for PDGF was stressed, in comparison with that of the relatively nonspecific Gleevec. The molecular role of PDGF in the development of blood vessels was also discussed, as were the effects of Gleevec in reducing interstitial fluid pressure in tumors with consequent facilitation of the uptake of certain drugs. Dr. G. Groner discussed the development and involution of breast as a model to study molecular regulations and interventions. The key role of the signal transducer and activator of transcription 5 was pointed out, with particular emphasis on the regulation of apoptosis and of the cell cycle. Suitable aptamers were indicated as agents interfering with various steps in signal transduction from a receptor to the nucleus. Dr. G. Groner also advocated the use of aptamers inserted into scaffold proteins as specific DNA binding agents and inhibitors acting at the dimerization domain of the signal transducer and activator of transcription 3 .

Intertwined with these four outstanding presentations by invited speakers from abroad were three interesting presentations by Dr. E. Kimura from the Instituto de Ciências Biomédicas, Universidade de São Paulo, Dr. G. Amarante-Mendes from the same Institute, and Dr. S. Saad from the Centro de Hematologia e Hemoterapia, Universidade Estadual de Campinas. Dr. E. Kimura discussed the impact of BRAF mutations in thyroid cancer and compared this tumor with the corresponding normal tissue also with reference to ras signal pathways. In thyroid papillary cancer mutations of genes coding for RET or TRK tyrosine kinases receptors were seen. BRAF somatic mutations were unique to this tumor and not found in other differentiated follicular thyroid cancer. The functional associations among Ras, RET and BRAF were discussed. BRAF mutations are also found in melanoma and in ovarian and colon cancer: the most frequent mutation is on exon 15. Dr. G. Amarante-Mendes discussed the mechanisms underlying the BcrAbl-mediated resistance to apoptosis. HL60 cells with ectopic expression of Bcr-Abl were compared with parent HL60 cells. BcrAbl protected the cells from apoptosis induced by several different agents. In protected cells, levels of Bcl-xL, Mcl-1 and Flip were increased compared to those in normal HL60 cells. These phenomena were not ob- 
served in other cell types such as SKW64 cells, suggesting that multiple factors may be involved in the protection from apoptotic agents. Dr. S. Saad discussed mechanisms of resistance to inhibitors of tyrosine kinases. A new inhibitor of Abl was discussed, as was the increased degradation of Bcr-Abl induced by 17AAG. The mechanisms involved in the action of mycophenolic acid and of OSU2012 were also analyzed as examples. In poster presentations this group also showed that the combined inhibition of mTOR and IRS was synergistic in reducing the growth of PC-3 prostate cells. Also, the discovery and function of the novel human gene ARGFAP21 was illustrated and the regulation of its expression by ROS-inducing agents outlined.

Session IV was dedicated to studies of tumor suppressor functions. Dr. M. Sogayar from the Instituto de Química, Universidade de São Paulo, outlined a search for new molecular targets that might contribute to tumor diagnosis and intervention. She used the rat ST1 cell line which is a C6 glioma cell very responsive to glucocorticoids. Using substraction DNA libraries a gene was isolated which is similar to human NRP/B. The ST1 cells were transfected with NRP/B constructs; hydrocortisone caused a reduction of colony cell formation from these cells with an indication that NRP/B may have a tumor suppression function. NIH-3T3 cells transfected with RECK show a slower rate of growth compared to controls and this was accompanied by retarded entry into the Sphase. This effect was related to a delay in $\mathrm{Rb}$ phosphorylation. In this and other models, RECK gene products also inhibited the action of matrix metalloproteases and reduced tumor invasion. Surprisingly, RECK was overexpressed in high grade gliomas, similarly to myc. Thus, expression of RECK was regulated during glioma and perhaps other tumor progression. This very interesting presentation indicated that this group is working at the cutting edge of investigations aimed at understanding molecular mechanisms of tumor progression and their reversal in glioma and other tumor types.

Dr. J. Silva, from the Universidade Federal do Rio de Janeiro, gave a detailed presentation on the structural biology of P53 aiming at identifying new targets for the development of new drugs. He showed evidence for different conformations of P53 and for hot-spot mutations, mainly in the DNA binding domain.

Dr. E. Rego, from the Faculdade de Medicina de Ribeirão Preto, Universidade de São Paulo, discussed molecular mechanisms of leukemogenesis focusing on acute myeloid leukemia and acute promyelocytic leukemia (APL). In $98 \%$ of the patients with APL the leukemia is associated with a 15-17 translocation which generates the promyelocytic leukemia (PML)-RAR $\alpha$ fusion gene; this oncoprotein interacts with PML and inhibits its anti-proliferation function. Translocations 11-17 generate PL2F-RAR $\alpha$ fusion proteins and leukemias with this marker are resistant to both ATRA and As $\mathrm{Cl} 3$. Transgenic hCG-PML-RAR $\alpha$ mice develop a leukemia similar to human APL after a long period of latency. Alterations of cell cycle control mechanisms were elegantly studied at different ages after birth and correlated with the onset of APL. It was also found that in the pre-leukemic stages, beyond 9 months of age, bone marrow cells were resistant to $\gamma$-irradiation-induced apoptosis and showed a block in G1/S. In a poster Dr. E. Rego's group outlined the antileukemic activity, in acute myeloid leukemia models, of a new alkylphospholipid, 10-oxa-octadecylphosphocholine, which promoted apoptosis and inhibited proliferation through interference with the phosphatidyl-inositol 3 kinase pathway.

Dr. T.A. Dragani from the Istituto Nazionale Tumori, Milan, Italy, gave a lecture focusing on the human relevance of models of mouse cancer modifier loci. The mouse model used lent itself to studies of the ge- 
netic predisposition to lung adenocarcinoma induced by urethane; C57BL/6J mice were resistant to genetically predisposed lung tumors if they possessed the multigene family tumor modifier Pas 1. An acute inflammatory response with a role in carcinogenesis is also genetically determined and is modulated by Pas 1 . Susceptibility to carcinogenesis is affected by the balance between Pas 1 and Pas 1-4. Also in humans cancer modifier genes affect susceptibility and response. Population-based association studies were discussed and the relationships of certain gene polymorphism to survival were elucidated comparing non-smokers lung patients less than 60 years old to a matched general population. This excellent presentation showed how a relevant mouse model can provide information guiding and enriching human experimentation.

Session V was focused on mechanisms of cell death and survival. Dr. R. Linden from the Universidade Federal do Rio de Janeiro, discussed targeting early events of apoptosis in the central nervous system. A model used was rat retina cells in which apoptosis is blocked by anisomycin inhibition effects. Nuclear exclusion of transcription factors such as those of MAX follows axon damage independent of caspase activity. After inhibition of protein synthesis not only MAX but also JUN and other transcription factors are excluded from the nucleus and this phenomenon as related to the development of apoptosis was discussed, among others.

Dr. C. Ferreira outlined the present status of targeted therapy of cancer and what was learned from translational studies. He proposed the possibility of starting attempts to materialize the dream of developing individualized chemotherapy based on knowledge being acquired in implementing targeted chemotherapy. He mentioned several examples of clinical progress in this area: monoclonal antibodies against appropriate growth factors or receptors, compounds like
Tarceva or ZD1839 with activity in squamous cell lung cancer, Gleevec for gastrointestinal stromal tumor, and others. $\mathrm{He}$ guarded against the empirical use of combinations, particularly those including wellknown cytotoxic agents. Indeed, attempts to select patients for a given targeted treatment based on the presence of the target in their tumors and their expected response to inhibitors of that target are becoming a current approach in modern chemotherapy and represent the beginning of the individualized chemotherapy projected by Dr. C. Ferreira for the future.

Session VI was dedicated to studies of tumor microenvironment. In this area at the cutting edge of tumor biology there were 4 presentations by Brazilian scientists: Dr. R. Borojevic from the Universidade Federal do Rio de Janeiro, Dr. J. Scharfstein from the same University, Dr. J. Chagas from the Universidade de Mogi das Cruzes in São Paulo, and Dr. M. Zago from the Faculdade de Medicina de Ribeirão Preto, Universidade de São Paulo. Dr. R. Borojevic discussed the microenvironment of tumor cells in the bone marrow. He emphasized that $10^{11}$ cells are produced every day. Bone marrow cells are around blood vessels and interact with bone cells. Mesenchymal stroma cells include reticular cells, fat storing cells, osteoblast endothelial cells, macrophages, and lymphocytes and are in intimate contact with hematopoietic cells. Several chemokines determine cell traffic inside the bone marrow. Gangliosides are present in the stroma and are involved in intercellular communications. The profiles of relevant genes in different groups of cells in the bone marrow were discussed. Dr. Scharfstein outlined the role of $\mathrm{G}$ protein coupled receptors in regulating the secretion of lysosomal proteases from prostate tumor cells. Leakage of proteases into the stroma provides a stimulus to dendritic cells (DC) and in turn favors DC-T effector cell interactions. Kinins have several physiological effects including the cau- 
sation of edema, vasodilatation and microvascular homeostasis. Kinin receptor signaling to DC and macrophages stimulates innate and adaptive immunity. In PC-3 and DU145, the excretion of various proteases as regulated by different kinases was competently discussed and their role in inflammation, immunity and tumor cell migration outlined. Dr. J. Chagas discussed serine protease inhibitors and their effects on prostate cancer. The activity of specific serpins as inhibitors of kallikrein hk2 and modifiers of human prostate cancer xenografts was outlined, with emphasis on specific hk2 pentapeptidic sequences. The human kallikrein gene is located on chromosome 19 and its product levels in blood are much lower than those of prostate-specific antigen, but hk2 hydrolyzes fibronectin, activates pro-prostate-specific antigen and causes the liberation of growth and angiogenic factors. These findings are consistent with an important role of the microenvironment in influencing the pathophysiology of tumors in the prostate milieu. Dr. M. Zago discussed abnormal gene expressions and their consequences in chronic lymphoproliferative disorders involving B cells or NK cells and in lymphoblastic lymphoma, chronic myeloid leukemia, B cell lymphoma, and Mantel cell lymphoma. Among the abhorrent genes are P13k/ akt, WNT, TGFß, and others. TGFß is increased in Mantel cell lymphoma and inhibitors of its pathway increase apoptosis. The selective expression of certain genes in some of the diseases considered was emphasized and provides evidence for distinct molecular individualities among different types of lymphoproliferative disorders.

The last day of the meeting started with a lecture by R. Gillies from the University of Arizona, USA, who discussed imaging of the tumor microenvironment and exploring its functions in relation to tumor progression and therapy. A key question he entertained was why cancer has a high aerobic glycolysis. Ninety percent of patient with metastatic cancer have evidence of high glycolysis in their tumor as evidenced by PET imaging. A number of proteins are degraded and glycolysis plus the action of HIF- $1 \alpha$ are essential for survival and angiogenesis. Tumor cells are under hypoxia selection but HIF- $1 \alpha$ is up-regulated even in the presence of oxygen in certain tumor models, but not in all. HIF$1 \alpha$ increases glycolysis slowly and this effect is greatly accelerated in the presence of c-Myc. Differences in $\mathrm{pH}$ have an effect on these phenomena and alkalinization leads to a decrease in spontaneous metastasis. Glycolysis helps cells to survive under metabolic pressures and these changes can now be monitored by the use of imaging technology.

Session VII dealt with tumor immunology and cancer vaccines. Dr. Villa's group at the Ludwig Institute for Cancer Research in São Paulo, is involved in molecular studies of the control of HPV infectivity and HPV action in cervical cells as well as in epidemiological studies of the preventive effects of HPV vaccines. This latter part of her activities was the major focus of her outstanding presentation. Problems in early diagnosis of cervical cancer through various types of screens were outlined and the fact that these problems are particularly serious in developing countries was emphasized. Sexual contacts of different kinds are responsible for infection. The infection rate is increased when sexual relations are started at a younger age. Prevention is implemented through abstinence and monogamy, and risk reduction is achieved by using condoms and among circumcised men. HPV-induced genital warts are also a problem in young people. The Merck vaccine Gardasil, which is based on virus particles as antigen, has low toxicity and induces high antibody responses. The vaccine should be given to children before the beginning of sexual activity and protection should last 10 years, with longer protection being still under study. It should be noted that after the age of 20, the majority of 
women have been exposed to HPV and are seropositive: they mount anamnestic responses to the vaccine. A reduction of at least $50 \%$ of cervical cancer can be expected to be obtained by the use of this vaccine.

Dr. A. Barbuto from the Instituto de $\mathrm{Ci}$ ências Biomédicas of the Universidade de São Paulo, discussed the important role of DC in cancer immunotherapy. After reviewing the role of different antigen-presenting cells in tumor immunity he focused on DC, indicating ways to obtain them in activated form in cell cultures with increased temperature. He emphasized the heterogeneity of the DC population and posed questions about which would be the desired phenotype of DC that would be effective in therapy. The utilization of DC fused with tumor cells as vaccines was discussed as was the vaccine dose dependence of antitumor responses.

The last session of the meeting was devoted to considerations of cancer biology and clinical oncology in the post-genomic era. Dr. L. Reis discussed esophageal and stomach cancer as examples. Using cDNA microarrays the expression profiles of more than 70 samples from normal and cancerous stomach, normal and Barrett esophagus as well as adenocarcinomas of the esophagus were defined. The expression profiles of 4800 genes were examined and functional modules of genes identified. Cytokine receptors, as a group, were increased in adenocarcinoma, glycerolipid pathways were decreased in adenocarcinoma and increased in non-malignant tissues. Clear separations between normal esophagus and esophagitis mucosa, tumor samples and metaplastic/columnar samples could be based on gene profiles. For example, cytokine receptor profiles discriminated between squamous and columnar tissue, c-kit was a marker for normal gastric mucosa, ILI R2 was augmented in metaplasia of stomach and esophagus mucosa, and so on. There is no doubt that the avant-garde studies of Dr. L. Reis represent a way of the future in the diagnosis of sub- groups of diseases which would provide powerful tools for the design of individualized treatments.

Dr. S. Verjovski-Almeida, from the Instituto de Química, Universidade de São Paulo, discussed intronic non-coding RNAs in cancer and pointed out that single gene tests are not as effective as multiple genes tests for the identification of patient subgroups and for the prediction of their response as well as the assessment of their therapeutic needs. The delineation of gene expression profiles could in fact discriminate, for example, between patients needing or not adjuvant chemotherapy. Gene expression signatures also allowed the identification of degree of malignancy of prostate cancer: this study is only 2 years old and follow-up evaluation is needed. Using the $\mathrm{LNCaP}$ model the effects of androgens on the expression of different groups of genes was studied and evidence was obtained for an androgen-dependent regulation of several gene profiles.

Dr. A. Camargo from the Ludwig Institute for Cancer Research in São Paulo discussed the role of ADAM 23 in breast cancer metastasis. ADAM 23 is a desintegrin and metalloprotease domain including a family of membrane-bound cell surface proteins with a shared structural organization. They have a major role in proteolysis, cell to cell and cell to matrix interactions and are important for tumor progression and the development of metastasis. However, ADAM 23 lacks a conserved metalloprotease domain, a fact suggesting that this compound has primarily a role in cell adhesion. Promoter hypermethylation occurs in $34 \%$ of advanced breast cancer cases. In the studies reported, RNAi reduced the expression of ADAM 23 and increased integrin $\alpha \beta 3$ activation in MDA-MB-435 cells, with increased cell affinity for vitronectin and increased migration. The results discussed suggest that ADAM 23 may be a new metastasis suppressor gene. 
At the end of the meeting, F. Rauscher, from the Wistar Institute in Philadelphia, USA, discussed the role of chromatin modifying enzymes in mediating gene silencing and their possible exploitation as new therapeutic targets in cancer. He described the role of chromatin structure and nuclear architecture in the control of gene expression: histone deacetylase (HDAC), histone acetyl transferase and histone methyl transferase all have a role in this regulation. It should be noted that HDACs have tissue specificity which might provide advantages for cancer chemotherapy. Questions were discussed as to whether histone modifications are interdependent and how the code is maintained. Targeting the $\mathrm{Zn}$ finger domain with DNAbinding specificity and coordination of silencing mechanisms, for example, with KAP co-repressor, were outlined. HDAC and KAP-1 form complexes which are affected by HDAC inhibitors and may be important for the function of KAP. The specificity in the function of methyl transferases and the recognition by heterochromatin protein of specific methyl modifications were outlined as implemented by direct binding to modified histones and linking to the co-repressor KAP-1. It is now possible to separate active regions from inhibited areas of the genome. This was an outstanding lecture discussing avant-garde aspects of mechanisms of gene expression and silencing.

In conclusion, as already indicated at the beginning of this commentary, this conference has not only contributed significantly to avant-garde knowledge in the areas discussed and provided an exciting educational opportunity to the young scientists who attended it, but also gave a very clear indication of the high levels of scientific expertise and activities in cancer research in Brazil. Certainly it is hoped that this worthy series of symposia will continue and will provide the same exciting opportunities for crossfertilization and advanced education that were provided by this symposium. It was a privilege to be able to participate in such an outstanding meeting. 